In Crescendo, 2017; 8(2): 255-264

Fecha de recepción: 2 de enero del 2017

Fecha de aceptación: 27 de octubre del 2017

\title{
NIVEL DE MADUREZ DE LA ALINEACIÓN, PLANIFICACIÓN Y ORGANIZACIÓN DE TIC EN LA ULADECH CATÓLICA USANDO COBIT 5
}

\author{
LEVEL OF MADURITY OF ALIGNMENT, PLANNING AND \\ ORGANIZATION OF INFORMATION AND COMUNICATION \\ TECHNOLOGY AT ULADECH CATOLICA USING COBIT 5
}

Jorge Luis Gutiérrez Gutiérrez'

\section{RESUMEN}

Esta investigación pertenece a la línea de investigación en Tecnologías de Información y Comunicación (TIC) de la Escuela Profesional de Ingeniería de Sistemas de la Universidad Católica los Ángeles de Chimbote, el cual determina en qué nivel de madurez de acuerdo al Cobit 5 se encuentran los procesos TIC en la ULADECH Católica. El estudio es de tipo descriptivo, no experimental, de corte transversal. La técnica de investigación utilizada es la encuesta y la entrevista; la población muestral está constituida por los responsables de la división de sistemas de la mencionada universidad. Los resultados han demostrado que los procesos TIC se encuentran el nivel 1 en la dimensión Alinear, Planificar y Organizar correspondiente al primer dominio de gestión de Cobit 5 .

PAlabras ClaVE: Procesos, tecnologías, Cobit.

1 Doctor en Ingeniería, conferencista internacional en Informática y Sistemas.jlgutgut@yahoo.es 


\begin{abstract}
This research is part of the research in information and communication technologies (ICT) of the Professional School of Systems Engineering at the Catholic University Angels of Chimbote, which determine the level of maturity of technologies processes based in Cobit 5 . The study is not experimental, descriptive and transversal time. The research technique used is the survey and interview; the sample population consists of chief of information at technogical division at this university. The results have shown that the processes are in level 1 in the first domain of management of Cobit 5.
\end{abstract}

KEY WORDS: Process, technologies, Cobit

\title{
INTRODUCCIÓN
}

La planeación y organización de TI es la fuente principal para lograr el objetivo de los negocios, COBIT está diseñado para ser utilizado no sólo por proveedores de servicios, usuarios y auditores de TI, sino también y principalmente, como guía integral para la gerencia y para los dueños de los procesos de negocio. Para proporcionar la información que la empresa requiere para lograr sus objetivos necesita invertir en TI, y administrar y controlar los recursos de TI usando un conjunto estructurado de procesos que provean los servicios que entregan la información empresarial requerida. El marco de trabajo $\mathrm{COBIT}^{1}$ ofrece herramientas para garantizar la alineación con los requerimientos del negocio (organización).

El plan estratégico de TI es necesario para gestionar y dirigir todos los recursos de tecnología de información en línea con la estrategia y prioridades del negocio (organización). En la actualidad muchas organizaciones no planifican la estrategia de TI como se debería, el cual es importante para lograr los objetivos del negocio, generar mayor ingreso, mantener costos bajos, mejorar el servicio al cliente en la organización e integrarse a la estrategia de la organización.

La arquitectura de la información es importante para determinar la estructura coherente de módulos que soportan la tecnología de información en la organización.

En cuanto a la determinación de la dirección tecnológica, la gerencia de sistemas o su equivalente debe estar consciente de la importancia del plan de infraestructura tecnológica. El proceso para el plan de infraestructura tecnológica es razonablemente sólido y está alineado con el plan estratégico de TI. 
La orientación de la infraestructura tecnológica incluye el entendimiento dónde la empresa desea ser líder y dónde desea rezagarse respecto al uso de tecnología, con base en los riesgos y en la alineación con la estrategia organizacional. Existen ciclos temporales donde el negocio se alinea a la tecnología o la tecnología se alinea al negocio. Los procesos, organización y relaciones de TI, en la organización deben tomar en cuenta los requerimientos del personal; debe existir la transparencia como el involucramiento de alto ejecutivo, gerencia del negocio, aseguramiento de calidad, administración de riesgo, seguridad de la información y garantizar los requerimientos del negocio

De acuerdo con Salazar ${ }^{2}$ las TI agregan valor a las actividades operacionales y de gestión empresarial en general y permite a las empresas obtener ventajas competitivas, permanecer en el mercado y centrarse en su negocio.

Varas ${ }^{3}$ aplica una encuesta basado en COBIT 4.0 a la empresa Acuapesca para determinar el perfil de tecnologías de información enfocándose a los procesos de planificación y organización.

Para las organizaciones es vital la existencia de una gran comunicación interna entre sus departamentos y externa con sus clientes y proveedores, además es importante que el flujo de información cada vez sea más rápido para que se puedan identificar los problemas en el menor tiempo posible y se puedan tomar decisiones oportunas para resolverlos, de manera que es necesario invertir en las TI, generando más beneficio para la empresa, mayores ingresos, procesos de negociación a corto plazo, comunicación con los proveedores rápida, confiable y con mayor seguridad, mejores servicios al cliente.

Gutiérrez $z^{4}$ hizo una medición de los procesos de la dimensión Planificar y Organizar de Cobit versión 4, obteniéndose como resultado que algunos procesos se encontraban en un nivel de madurez repetido y otros en un nivel definido.

Esta investigación se justifica porque permite aplicar el modelo de evaluación de procesos (PAM, por sus siglas en inglés) de COBIT 5 y determinar así en qué nivel se encuentran los procesos de TI de la ULADECH Católica respecto de la dimensión Alinear, Planificar y Organizar.

En esta investigación se enuncia el siguiente problema de investigación ¿Cuál es el nivel de madurez de los procesos TI de la ULADECH Católica en el año 2016 de acuerdo al marco COBIT 5? Se postula como hipótesis: el nivel de madurez de los procesos TI de la UALDECH Católica se encuentra en el nivel 2 de acuerdo al marco COBIT 5. 
El objetivo general que se formula es determinar el nivel de madurez de los procesos de tecnologías de la información en la ULADECH Católica en el año 2016 de acuerdo al marco COBIT 5; y los objetivos específicos que se formulan son: describir los procesos de TI de la ULADECH Católica correspondientes al marco COBIT 5, aplicar un instrumento para determinar el nivel de madurez de los procesos y analizar los resultados proponiendo recomendaciones para la mejora continua.

\section{MATERIAL Y MÉTODO}

\section{TiPO DE INVESTIGACIÓN}

En esta investigación se aplica el método científico inductivo, puesto que se estudia los procesos TI de la dimensión Alinear, Planificar y Organizar y luego se tratará de generalizarlas para todas las dimensiones en la ULADECH Católica. Se afirma que con resultados parciales en la muestra de estudio se generaliza a toda la población.

\section{DISEÑO DE INVESTIGACIÓN}

Investigación de tipo cualitativo, diseño no experimental, de tipo descriptivo, de corte transversal y se grafica de la siguiente manera:

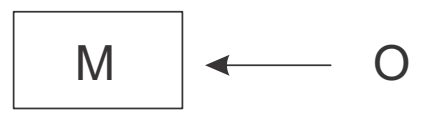

Donde:

M: Muestra

O: Observación

\section{POBLACIÓN Y MUESTRA}

La población está constituida por los procesos de las 5 dimensiones del modelo Cobit 5 correspondientes a la ULADECH Católica. La muestra está constituida por una dimensión, la de Alinear, Planificar y Organizar que es la primera dimensión del dominio gerenciar. Se utilizó el muestreo no probabilístico, decidiendo las áreas de la muestra de acuerdo a las facilidades de acceso a información. 


\section{TÉCNICAS E INSTRUMENTOS UTILIZADOS}

Se aplicó una encuesta basada en el modelo de evaluación de procesos, (PAM, por sus siglas en inglés), y se retroalimentó las respuestas de la encuesta con una entrevista al jefe de la división de sistemas de la ULADECH Católica.

Tabla 1

ENCUESTA SEGÚN PAM PARA MEDIR LA MADUREZ DE LOS PROCESOS TI

\begin{tabular}{ccl}
\hline \multicolumn{1}{c}{ Nivel } & \multicolumn{1}{c}{ Pregunta } & Respuesta \\
\hline \multicolumn{4}{c}{1} & 1.1 & Hay evidencia de que una práctica básica está siendo ejecutada. \\
& 1.2 & $\begin{array}{l}\text { Los productos de trabajo (Work Products) demuestran evidencia de que } \\
\text { el proceso tiene las salidas esperadas. }\end{array}$ \\
\hline
\end{tabular}

2.1 Objetivos de la performance del proceso son identificados.

2.2 La performance del proceso es planeada y monitoreada.

2.3 La performance del proceso es ajustada para alcanzar planes.

2.4 Las responsabilidades y autoridades para ejecutar el proceso son definidas, asignadas y comunicadas.

2.5 Los recursos y la información necesaria para ejecutar el proceso son identificadas.

2.6 Interfaces entre las partes involucradas son gestionadas para asegurar una comunicación efectiva y una clara asignación de responsabilidades.

2.7 Requerimientos para los productos de trabajo (work product) de cada proceso son definidos.

2.8 Los requerimientos para el control y la documentación de los productos de trabajo son definidos.

2.9 Los productos de trabajo son definidos documentados y controlados.

3.1 Un proceso estándar incluyendo guías es definido para describir los elementos fundamentales que deben ser incorporados dentro del proceso.

3.2 Se determina la secuencia de interacción del proceso estándar con otros procesos.

33.3 Competencias requeridas y roles para ejecutar procesos son identificados como parte del proceso.

3.4 Infraestructura y entorno de trabajo requerido son identificados como parte del proceso estándar.

3.5 Métodos adecuados para monitorear la efectividad y la adecuabilidad de los procesos son determinados. 


\section{PROCESAMIENTO DE RECOLECCIÓN Y ANÁLISIS DE DATOS}

Se describe inicialmente los procesos de la ULADECH Católica, luego se aplica una encuesta basada en el PAM, obteniéndose el cumplimiento o no de cada una de los ítems propuestos organizados por 13 factores correspondientes al dominio de gestión Alinear, Planificar y Organizar de Cobit 5; finalmente se valida los resultados con una entrevista de retroalimentación al jefe de la división de sistemas.

\section{RESULTADOS}

En base a que la mayoría de empresas no han alcanzado el nivel 5 de madurez, se ha delimitado el contenido del instrumento para medir hasta el nivel 3; además se ha considerado de acuerdo a la muestra la dimensión Alinear, Planificar y Organizar correspondiente al dominio gestión de TI de Cobit 5.

Se ha determinado el nivel de madurez de acuerdo a 13 procesos:

\section{Gestionar el marco de gestión de TI}

Aclarar y mantener el gobierno de la misión y la visión corporativa de las TI. Implementar y mantener mecanismos y autoridades para la gestión de la información y el uso de las TI en la empresa, para apoyar los objetivos de gobierno en consonancia con las políticas y los principios rectores.

\section{Gestionar la estrategia}

Proporcionar una visión holística del negocio actual y del entorno de TI, la dirección futura, y las iniciativas necesarias para migrar al entorno deseado. Aprovechar los bloques y componentes de la estructura empresarial, incluyendo los servicios externalizados y las capacidades relacionadas que permitan una respuesta ágil, confiable y eficiente a los objetivos estratégicos.

\section{Gestionar la arquitectura empresarial}

Establecer una arquitectura común compuesta por los procesos de negocio, la información, los datos, las aplicaciones y las capas de la arquitectura tecnológica de manera eficaz y eficiente para la realización de las estrategias de la empresa y de las TI, mediante la creación de modelos clave y prácticas que describan las líneas de partida y las arquitecturas objetivo. Definir los requisitos para la taxonomía, las normas, las directrices, los procedimientos, las plantillas y las herramientas y proporcionar un vínculo para estos componentes. Mejorar la adecuación, aumentar la agilidad, mejorar la calidad de la información y generar ahorros de 
costes potenciales mediante iniciativas tales como la reutilización de bloques de componentes para los procesos de construcción.

\section{Gestionar la innovación}

Mantener un conocimiento de la tecnología de la información y las tendencias relacionadas con el servicio, identificar las oportunidades de innovación y planificar la manera de beneficiarse de la innovación en relación con las necesidades del negocio. Analizar cuáles son las oportunidades para la innovación empresarial o qué mejora puede crearse con las nuevas tecnologías, servicios o innovaciones empresariales facilitadas por TI, así como a través de las tecnologías ya existentes y por la innovación en procesos empresariales y de las TI. Influir en la planificación estratégica y en las decisiones de la arquitectura de empresa.

\section{Gestionar el portafolio}

Ejecutar el conjunto de direcciones estratégicas para la inversión alineada con la visión de la arquitectura empresarial, las características deseadas de inversión, los portafolios de servicios relacionados, considerar las diferentes categorías de inversión y recursos y las restricciones de financiación.

\section{Gestionar el presupuesto y los costes}

Gestionar las actividades financieras relacionadas con las TI tanto en el negocio como en las funciones de las TI, abarcando presupuesto, coste y gestión del beneficio, y la priorización del gasto mediante el uso de prácticas presupuestarias formales y un sistema justo y equitativo de reparto de costes a la empresa. Consultar a las partes interesadas para identificar y controlar los costes totales y los beneficios en el contexto de los planes estratégicos y tácticos de las TI, e iniciar acciones correctivas cuando sea necesario.

\section{Gestionar los Recursos Humanos}

Proporcionar un enfoque estructurado para garantizar una óptima estructuración, ubicación, capacidades de decisión y habilidades de los recursos humanos. Esto incluye la comunicación de las funciones y responsabilidades definidas, la formación y planes de desarrollo personal y las expectativas de desempeño, con el apoyo de gente competente y motivada.

\section{Gestionar las relaciones}

Gestionar las relaciones entre el negocio y TI de modo formal y transparente, enfocándolas hacia el objetivo común de obtener resultados empresariales 
exitosos, apoyando los objetivos estratégicos y dentro de las restricciones del presupuesto y los riesgos tolerables. Basar la relación en la confianza mutua, usando términos entendibles, lenguaje común y voluntad de asumir la propiedad y responsabilidad en las decisiones claves.

\section{Gestionar los acuerdos de servicio}

Alinear los servicios basados en TI y los niveles de servicio con las necesidades y expectativas de la empresa, incluyendo identificación, especificación, diseño, publicación, acuerdo y supervisión de los servicios TI, niveles de servicio e indicadores de rendimiento.

\section{Gestionar los Proveedores}

Administrar todos los servicios de las TI prestados por todo tipo de proveedores para satisfacer las necesidades del negocio, incluyendo la selección de los proveedores, la gestión de las relaciones, la gestión de los contratos y la revisión y supervisión del desempeño, para una eficacia y cumplimiento adecuados.

\section{Gestionar la calidad}

Definir y comunicar los requisitos de calidad en todos los procesos, procedimientos y resultados relacionados de la organización, incluyendo controles, vigilancia constante y el uso de prácticas probadas y estándares de mejora continua y esfuerzos de eficiencia.

\section{Gestionar el riesgo}

Identificar, evaluar y reducir los riesgos relacionados con TI de forma continua, dentro de niveles de tolerancia establecidos por la dirección ejecutiva de la empresa.

\section{Gestionar la seguridad}

Definir, operar y supervisar un sistema para la gestión de la seguridad de la información.

En la tabla 2 se pueden apreciar los resultados que la encuesta aplicada arroja para cada proceso.

En la tabla 3 se muestra el nivel de madurez medido de acuerdo al nivel de logro de los atributos de los procesos TI en la ULADECH Católica. 
Tabla 2

RESULTADOS DE LA ENCUESTA APLICADA A 13 PROCESOS DE LA DIMENSIÓN ALINEAR, PLANIFICAR Y ORGANIZAR

\begin{tabular}{|c|c|c|c|c|c|c|c|c|c|c|c|c|c|c|}
\hline Nivel & Pregunta & 1 & 2 & 3 & 4 & 5 & 6 & 7 & 8 & 9 & 10 & 11 & 12 & 13 \\
\hline \multirow{2}{*}{1} & 1.1 & $\mathrm{~s}$ & s & s & s & $\mathrm{s}$ & s & S & s & $\mathrm{s}$ & $\mathrm{s}$ & $\mathrm{s}$ & $n$ & $\mathrm{~s}$ \\
\hline & 1.2 & $\mathrm{~s}$ & s & s & $n$ & $\mathrm{~s}$ & s & S & s & $\mathrm{s}$ & s & $\mathrm{n}$ & $\mathrm{n}$ & s \\
\hline \multirow{9}{*}{2} & 2.1 & $\mathrm{~s}$ & s & s & s & $\mathrm{s}$ & s & S & s & $\mathrm{s}$ & s & $\mathrm{n}$ & $\mathrm{n}$ & s \\
\hline & 2.2 & $\mathrm{~s}$ & $\mathrm{n}$ & $n$ & s & $\mathrm{s}$ & s & s & s & $\mathrm{s}$ & s & $\mathrm{n}$ & $n$ & $\mathrm{~s}$ \\
\hline & 2.3 & $\mathrm{n}$ & s & s & s & $\mathrm{S}$ & s & S & s & $n$ & $n$ & $n$ & $n$ & $\mathrm{~s}$ \\
\hline & 2.4 & $\mathrm{~s}$ & s & s & s & $\mathrm{S}$ & $n$ & S & s & $n$ & $n$ & $n$ & $n$ & $\mathrm{~s}$ \\
\hline & 2.5 & $\mathrm{n}$ & $\mathrm{n}$ & $\mathrm{n}$ & $\mathrm{n}$ & $n$ & $\mathrm{n}$ & $\mathrm{n}$ & $\mathrm{n}$ & $\mathrm{n}$ & $n$ & $\mathrm{n}$ & $\mathrm{n}$ & $\mathrm{n}$ \\
\hline & 2.6 & $n$ & $n$ & $n$ & $n$ & $n$ & $n$ & $n$ & $n$ & $n$ & $n$ & $n$ & $n$ & $n$ \\
\hline & 2.7 & $\mathrm{n}$ & $n$ & $\mathrm{n}$ & $\mathrm{n}$ & $\mathrm{n}$ & $n$ & $\mathrm{n}$ & $n$ & $\mathrm{n}$ & $n$ & $\mathrm{n}$ & $n$ & $\mathrm{n}$ \\
\hline & 2.8 & $\mathrm{n}$ & $n$ & $n$ & $n$ & $n$ & $n$ & $n$ & $n$ & $n$ & $n$ & $n$ & $n$ & $n$ \\
\hline & 2.9 & $\mathrm{n}$ & $n$ & $n$ & $n$ & $\mathrm{n}$ & $n$ & $\mathrm{n}$ & $n$ & $\mathrm{n}$ & $n$ & $\mathrm{n}$ & $n$ & $n$ \\
\hline \multirow{5}{*}{3} & 3.1 & $\mathrm{n}$ & $\mathrm{n}$ & $n$ & $n$ & $\mathrm{n}$ & $\mathrm{n}$ & $n$ & $n$ & $\mathrm{n}$ & $n$ & $n$ & $n$ & $\mathrm{n}$ \\
\hline & 3.2 & $\mathrm{n}$ & $\mathrm{n}$ & $n$ & $\mathrm{n}$ & $\mathrm{n}$ & $\mathrm{n}$ & $\mathrm{n}$ & $n$ & $\mathrm{n}$ & $n$ & $\mathrm{n}$ & $n$ & $\mathrm{n}$ \\
\hline & 3.3 & $\mathrm{n}$ & $\mathrm{n}$ & $\mathrm{n}$ & $\mathrm{n}$ & $\mathrm{n}$ & $\mathrm{n}$ & $\mathrm{n}$ & $\mathrm{n}$ & $\mathrm{n}$ & $n$ & $\mathrm{n}$ & $\mathrm{n}$ & $\mathrm{n}$ \\
\hline & 3.4 & $\mathrm{n}$ & $\mathrm{n}$ & $\mathrm{n}$ & $\mathrm{n}$ & $\mathrm{n}$ & $\mathrm{n}$ & $\mathrm{n}$ & $\mathrm{n}$ & $\mathrm{n}$ & $n$ & $\mathrm{n}$ & $\mathrm{n}$ & $\mathrm{n}$ \\
\hline & 3.5 & $\mathrm{n}$ & $\mathrm{n}$ & $\mathrm{n}$ & $\mathrm{n}$ & $\mathrm{n}$ & $\mathrm{n}$ & $\mathrm{n}$ & $n$ & $\mathrm{n}$ & $n$ & $n$ & $\mathrm{n}$ & $\mathrm{n}$ \\
\hline
\end{tabular}

Tabla 3

NIVEL DE MADUREZ POR PROCESO TI, RATING PORCENTUAL

\begin{tabular}{|c|c|c|c|c|c|c|c|c|c|c|c|c|c|c|}
\hline Nivel & $\begin{array}{c}\text { Atributos } \\
\text { de procesos }\end{array}$ & 1 & 2 & 3 & 4 & 5 & 6 & 7 & 8 & 9 & 10 & 11 & 12 & 13 \\
\hline 1 & AP 1.1 & 100 & 100 & 100 & 50 & 100 & 100 & 100 & 100 & 100 & 100 & 50 & 0 & 100 \\
\hline 2 & $\begin{array}{l}\text { AP } 2.1 \\
\text { AP } 2.2\end{array}$ & 33 & 33 & 33 & 44 & 44 & 33 & 44 & 44 & 22 & 22 & 0 & 0 & 44 \\
\hline 3 & $\begin{array}{l}\text { AP } 3.1 \\
\text { AP } 3.2\end{array}$ & 0 & 0 & 0 & 0 & 0 & 0 & 0 & 0 & 0 & 0 & 0 & 0 & 0 \\
\hline 4 & $\begin{array}{l}\text { AP } 4.1 \\
\text { AP } 4.2\end{array}$ & 0 & 0 & 0 & 0 & 0 & 0 & 0 & 0 & 0 & 0 & 0 & 0 & 0 \\
\hline 5 & $\begin{array}{l}\text { AP } 5.1 \\
\text { AP } 5.2\end{array}$ & 0 & 0 & 0 & 0 & 0 & 0 & 0 & 0 & 0 & 0 & 0 & 0 & 0 \\
\hline
\end{tabular}




\section{DISCUSIÓN}

De los resultados se concluye que los procesos TI de la ULADECH Católica se encuentran en el nivel 1, puesto que existen evidencias de que una práctica básica se está ejecutando como la de identificar y catalogar los servicios de TI, además los productos de trabajo demuestran que los procesos encuestados tienen las salidas esperadas. Sin embargo, hay varios procesos que todavía no han sido desplegados como el de gestionar el presupuesto y los costes, gestionar las relaciones, gestionar la calidad, y gestionar los riesgos.

Al contrastar los resultados con los antecedentes de la última medición ${ }^{4}$ se puede advertir que con el nuevo modelo los procesos TI de la ULADECH Católica se encuentran en un nivel ejecutado-nivel 1-.

\section{CONCLUSIONES}

1. Los procesos TI de la ULADECH Católica correspondientes a la dimensión Alinear, Planificar y Organizar de acuerdo a Cobit 5, se encuentran en su mayoría-11 de 13-en un nivel 1, nivel ejecutado.

2. Algunos procesos tienen cierta madurez, pero no se pueden considerar estar en un nivel 2, nivel Administrado; toda vez que no están largamente (> 50\%) o completamente (> 85\%) alcanzados.

3. Se concluye además que en contraste con la medición hecha anteriormente con Cobit 4, la madurez ha disminuido, recomendándose buscar un alto compromiso con la gerencia corporativa, e integrándose en una visión holística de procesos a nivel de toda la Universidad.

\section{REFERENCIAS BIBLIOGRÁFICAS}

1. TI Governance Institute. Objetivos de Control para la información y Tecnologías relacionadas (COBIT, en inglés: Control Objectives for Information and Related Technology) [Archivo en Internet]. United States of America: TI Governance Institute. 2007 [Citada 05 de Octubre 2015] Disponible desde: http://cs.uns.edu.ar/ ece/auditoria/cobiT4.1spanish.pdf

2. Salazar C. Las TIC como herramienta a la gestión empresarial. [Artículo en Internet]. Chile. Universidad Austral de Chile, Instituto Académico de Administración. 2008. [Citada 05 de Octubre 2015]. Disponible desde: http://cibermundos.bligoo.com/content/view/145501.

3. Varas P. Perfil de planeamiento y organización de Tecnologías de la Información y Comunicación (TIC) en la empresa Acuapesca SAC de la provincia de Casma.[Tesis para optar el título de ingeniero de sistemas]. Chimbote: Universidad Católica Los Ángeles de Chimbote; [citada 01 Enero 2016]. 2009.

4. Gutiérrez, J. Perfil de la planificación y organización de las Tecnologías de Información y Comunicaciones. Perú: In Crescendo Vol 4 N²: pp. 269-283; 2013.

5. Hernández, R., Fernández, C., y Baptista, P. Metodología de la Investigación (6ta ed.). México: Mc Graw Hill Interamericana; 2015. 